\title{
Can machine learning spin straw into gold?
}

\author{
Rittu Hingorani, MD, a and Christopher L. Hansen, MD, FASNC ${ }^{\mathrm{a}, \mathrm{b}}$ \\ a Thomas Jefferson University, Philadelphia, PA \\ b Jefferson Heart Institute, Philadelphia, PA
}

Received Mar 3, 2017; accepted Mar 3, 2017

doi: $10.1007 / \mathrm{s} 12350-017-0848-5$

\section{See related article, pp. 1601-1609}

"If you torture the data enough, nature will always confess",

-Ronald Coase

Almost from its inception, Nuclear Cardiology has depended upon computers. First, they were used for the simple act of image acquisition, then filtering, and now ever more complex methods of SPECT reconstruction. Much of this progress has been driven by the ongoing revolution in computing that is resulting in increasingly faster computers with greater memory at diminishing cost. This explosion in fast, cheap, computational power has permeated all aspects of medicine and society in general. The promises of "Big Data' and "'artificial intelligence" or "machine learning", to solve previously intractable problems are enticing and a continuing focus of research. Computers are being used to help answer many questions in science and medicine that until now have been difficult to approach and analyze. From genomics to drug discoveries and spatial mapping of various organs particularly in the field of neuroanatomy or electrophysiology to predictive medicine, computers are being relied upon more so than ever to help guide modern medicine. ${ }^{1,2}$ Many studies have looked at neural networks in cardiology to help solve problems in areas including coronary artery disease, electrophysiology, cardiac image analysis, and cardiac drug dosing. ${ }^{3}$ The first medical application of an artificial neural network (ANN) was by Hart in 1989 where he reported modest success. ${ }^{4}$ Baxt later reported good accuracy in the ability to diagnose acute myocardial infarction. ${ }^{5}$ Despite these

Reprint requests: Christopher L. Hansen, MD, FASNC, Jefferson Heart Institute, 925 Chestnut St, Mezzanine, Philadelphia, PA, 19107; christopher.hansen@jefferson.edu

J Nucl Cardiol 2018;25:1610-2.

1071-3581/\$34.00

Copyright (C) 2017 American Society of Nuclear Cardiology. early reports, ANNs have not yet found widespread clinical acceptance.

The study by Isma'eel, et al, in this volume of the Journal looks at the ability of an ANN to predict the presence of coronary artery disease (CAD) from clinical parameters in patients presenting to their stress lab. ${ }^{6}$ The results are compared to predictions developed by Diamond \& Forrester and then refined by Morise. ${ }^{7,8}$ ANNs are so named due to their similarity to biological neural networks: Successive layers of nodes allow for complex interactions between parameters giving the network the potential for discovering hidden patterns in the data. In fact, ANNs can exactly or almost exactly fit any dataset presented to them, a process known as overfitting.

Overfitting is not a problem unique to ANNs or machine learning. It is a frequent problem in general statistical modeling as well and has led to such general rules of thumb as having 10 times as many samples as parameters in the model and only the cautious use of polynomials. A common strategy to avoid this is the holdout method and was employed by the authors. The nomenclature may be confusing due to the terms the authors used here. In the holdout method, the data are separated into independent training (which the authors called derivation) and validation (which the authors named testing) sets. In this study, the authors used the term validation set for a subset of the derivation set. The training set is used to fit the models; the validation (testing) set is used to validate model selection; and the test set is used for the assessment of the generalization error of the final chosen model. ${ }^{9}$ Other techniques such as cross-validation, k-fold cross-validation, or Bootstrapping have been suggested as well, and it is suggested in the literature that k-fold cross-validation can potentially be a preferred method to assess generalizability especially with a $\mathrm{k}$ value of 20 or higher. ${ }^{10}$

The literature reminds us that neural networks are prone to overfitting. A problem that is worse with small training samples since smaller samples have higher variance. ${ }^{11}$ The authors had only 60 patients with CAD that then had to be divided between the training and validation sets. The k-fold cross-validation technique is 
another method to estimate the prediction error and is basically the holdout method repeated $\mathrm{k}$ times. As the value of $\mathrm{k}$ increases the variance decreases. It has been reported that the holdout method does worse than k-fold cross-validation to prevent overfitting. ${ }^{12}$

Toying with their model provides results that are suggestive of overfitting. ${ }^{13}$ For example, a 55-year-old woman who is diabetic and post-menopausal with typical angina is ischemic if she has pain in the upper right and mid-left chest (sectors $1 \& 6$ ) alone, but non-ischemic if the center of the chest (sector 5) is also involved. A sixty-year-old male with hyperlipidemia and atypical right-sided (sector 4) chest pain is ischemic, but with anginal chest pain is non-ischemic. These examples do not make sense clinically and suggest that the model suffers from overfitting. That is, the model is mimicking idiosyncrasies of the training set and not representing a meaningful physiologic relationship.

How much training data does one require? It is often difficult to provide a general rule on how much training data are enough; among other things, this depends on the signal-to-noise ratio of the underlying function and the complexity of the models being fit to the data. ${ }^{9}$

The authors mention ANN bootstrapping as a means to assign measure of accuracy, but it is unclear as to the type of bootstrapping used. Often, ANN bootstrap methods assume independent random samples that are free of outliers. However, if there is an existence of outliers in the sampling set, the subsequent datasets used for bootstrapping may have a higher level of contamination than the original dataset affecting the model adversely and leading to poor performance by the model. ${ }^{14}$

External validation is another important aspect that needs to be assessed before considering the applicability of such a model in medical practice. This is recognized by the authors and it is a potential cause for error because the dataset is small, and hence the training set is small which can affect the true generalizability of the results as obtained by the authors. Their model needs to be tested against wider population subsets before its generalizability can be established. It would have been helpful to assess the in-sample and out-of-sample figures in the study to evaluate the true performance of the network.

Angus noted that a controlling theme in studies related to model selection is the determination of a measure of prediction accuracy, or model fit, that takes into account both prediction variance and prediction bias, with the former tending to increase with model complexity (e.g., the number of terms in the linear regression function) and the latter tending to decrease with model complexity. ${ }^{15}$ Based on a training sample, the "best" model is then selected to achieve some optimum balance between these competing quantities as estimated in some fashion from the sample. This fact is echoed in other studies and states that the model should be selected based on its accuracy and performance using the cross-validation set and then the test set used once on this selected model. It appears the authors may have overfit their data. While the problem of overfitting in model selection is of the same nature as that of overfitting at the first level of inference, the lack of mathematical tractability appears to have limited the theoretical analysis of model selection via optimization of a model selection criterion. ${ }^{16}$ This brings us to another important pitfall called data snooping.

Data snooping is a loosely defined term sometimes called data dredging, data mining, or p-hacking. This can occur when a data asset is used more than once for selecting an appropriate model. A more focused interpretation of data snooping involves how the validation set is used. In the current study, the derivation set consisted of 60 patients, of which 48 were used to derive the model and the remaining 12 to assess its accuracy with the best model used on the validation (authors' term: testing) set. The problem arises when they tested 200 different models against the validation (testing) set. This challenges the maxim: "If a data set has affected any step in the learning process, its ability to assess the outcome has been compromised." 17 This, in effect, makes the validation (testing) set part of the training process and leaves the authors with no unbiased dataset with which to test their model.

ANNs behave much more like a "black box" than conventional statistical models. That is, the final model provides only the output. The relative importance of different parameters, their interactions, whether a relationship is linear, logarithmic, etc. is difficult to discern from the final model. A statistical model will require more work from and refinement by the investigator, but these efforts will be rewarded by greater insight into the underlying processes. Sargent reviewed the performance of ANNs compared to conventional statistical techniques in 28 different studies of medical issues and concluded that neither was ideal; they were in many ways complementary and ANNs should not replace conventional statistical methods. ${ }^{18}$

So, can Machine Learning spin straw into gold? Machine learning algorithms or even conventional statistical models (given enough parameters) can exactly reproduce any dataset presented to them. Investigators must take great pains to ensure that this represents the discovery of a true "hidden pattern" and not overfitting the data and mimicking the noise on top of a simpler underlying relationship. More importantly, data snooping must be avoided; it can be quite insidious and 
overlooked. Even abandoning one machine learning algorithm for another because of poor initial results can invalidate the results if the same validation set is used. In this instance, the validation set has now become part of the training process because it was used to reject one algorithm and choose another. Nevertheless, continued growth of "Big Data," machine learning, and raw computational power will combine to provide an endless source of hammers in search of clinical nails. We should continue to explore this potential and welcome new studies in exploring them. As clinicians, we need to understand the potential pitfalls and weaknesses of these new technologies, and maintain a healthy dose of skepticism before employing them in clinical practice. To answer the question: No, only Rumpelstiltskin can spin straw into gold, but we will never stop trying.

\section{Disclosure}

The authors declare that they have no conflict of interest.

\section{References}

1. Ermentrout B. Neural networks as spatio-temporal pattern-forming systems. Rep Prog Phys. 1998;61:353.

2. Prasad G.K, Sahambi J (2003) Classification of ECG arrhythmias using multi-resolution analysis and neural networks. In: IEEE conference on convergent technologies for the Asia-Pacific region (TENCON).

3. Itchhaporia D, Snow PB, Almassy RJ, Oetgen WJ. Artificial neural networks: Current status in cardiovascular medicine. J Am Coll Cardiol. 1996;28:515-21.

4. Hart A, Wyatt J (1989) Connectionist models in medicine: An investigation of their potential. In: Conference on Artificial Intelligence in Medicine (AIME 89). Springer, New York, pp. 115-124.

5. Baxt WG. Use of an artificial neural network for the diagnosis of myocardial infarction. Ann Intern Med. 1991;115:843-8.
6. Isma'eel HA, Sakr GE, Serhan M, Lamaa N, Hakim A, Cremer P et al (2017) Artificial neural network-based model enhances risk stratification and reduces non-invasive cardiac stress imaging compared to Diamond-Forrester and Morise risk assessment models: A prospective study. J Nucl Cardiol. pp. 1-9.

7. Diamond GA, Forrester JS. Analysis of probability as an aid in the clinical diagnosis of coronary-artery disease. $\mathrm{N}$ Engl $\mathrm{J}$ Med. 1979;300:1350-8.

8. Morise AP, Detrano R, Bobbio M, Diamond GA. Development and validation of a logistic regression-derived algorithm for estimating the incremental probability of coronary artery disease before and after exercise testing. J Am Coll Cardiol. 1992;20:1187-96.

9. Hastie T, Tibshirani RI, Frieman J (eds) (2009) The elements of statistical learning: Data mining, inference, and prediction. Wiley, New York

10. Kohavi R (1995) A study of cross-validation and bootstrap for accuracy estimation and model selection. In: International conference on artificial intelligence (IJCAI). Stanford, CA.

11. Samarasinghe $S$ (2016) Neural networks for applied sciences and engineering: From fundamentals to complex pattern recognition. CRC Press, Boca Raton.

12. Blum A, Kalai A, Langford J (1999) Beating the hold-out: Bounds for $\mathrm{k}$-fold and progressive cross-validation. In: Proceedings of the twelfth annual conference on computational learning theory. ACM, New York.

13. http://www.aub.edu.lb/fm/vmp/Documents/StressImaging/index. html. Accessed 28 Feb 2017

14. Allende H, Nanculef R, Salas R (2004) Robust bootstrapping neural networks. In: Mexican International conference on artificial intelligence. Springer, New York.

15. Angus JE (1991) Criteria for choosing the best neural network: Part 1. DTIC Document.

16. Cawley GC, Talbot NL. On over-fitting in model selection and subsequent selection bias in performance evaluation. J Mach Learn Res. 2010;11:2079-107.

17. Abu-Mostafa YS Learning from data: Lecture 17 three learning principles. http://work.caltech.edu/slides/slides17.pdf. Accessed 28 Feb 2017

18. Sargent DJ. Comparison of artificial neural networks with other statistical approaches: Results from medical data sets. Cancer. 2001;91:1636-42. 